\title{
Ghost-free vector superfield actions in supersymmetric higher-derivative theories
}

\author{
Toshiaki Fujimori, ${ }^{a}$ Muneto Nitta, ${ }^{a}$ Keisuke Ohashi, ${ }^{a}$ Yusuke Yamada $^{b}$ and \\ Ryo Yokokura ${ }^{c}$ \\ ${ }^{a}$ Department of Physics \& Research and Education Center for Natural Sciences, Keio University, \\ Hiyoshi 4-1-1, Yokohama, Kanagawa 223-8521, Japan \\ ${ }^{b}$ SITP and Department of Physics, Stanford University, \\ Stanford, CA 94305, U.S.A. \\ ${ }^{c}$ Department of Physics, Keio University, \\ Hiyoshi 3-14-1, Yokohama, Kanagawa 223-8522, Japan \\ E-mail: toshiaki.fujimori018@gmail.com, nitta@phys-h.keio.ac.jp, \\ keisuke084@gmail.com, yusukeyy@stanford.edu, \\ ryokokur@rk.phys.keio.ac.jp
}

ABstRACT: We systematically construct ghost-free higher-derivative actions of Abelian vector supermultiplets in four-dimensional $\mathcal{N}=1$ global supersymmetric theories. After giving a simple example which illustrates that a naive introduction of a higher-derivative term gives rise to a ghost, we discuss possible building blocks for a ghost-free action and explicitly show that their bosonic parts have no ghost mode and the auxiliary field $\boldsymbol{D}$ does not propagate. Higher-derivative terms yield higher powers of the auxiliary field $\boldsymbol{D}$ in the actions, and the D-term equations of motion consequently admit multiple solutions in general. We confirm that the well-known supersymmetric Dirac-Born-Infeld action falls into this class. We further give another example in which the standard quadratic kinetic term (Maxwell term) is corrected by a quartic term of the field strength. We also discuss possible couplings to matter fields and a deformed D-term potential.

KeYwords: Supersymmetric Effective Theories, Supersymmetric Gauge Theory

ARXIV EPRINT: 1708.05129 


\section{Contents}

1 Introduction 1

2 A higher-derivative action for a vector multiplet with a ghost 3

3 Ghost-free higher-derivative actions for vector multiplet $\quad 4$

3.1 The building blocks of ghost-free higher-derivative terms 5

$\begin{array}{lll}3.2 & \text { Examples of ghost-free higher-derivative action for vector multiplet } & 7\end{array}$

4 A coupling to matter fields $\quad 10$

$\begin{array}{llr}5 & \text { Summary and discussion } & 10\end{array}$

$\begin{array}{ll}\text { A Convention for vector superfield } & 11\end{array}$

\section{Introduction}

Higher-derivative terms beyond renormalizable ones inevitably appear in effective theories of fundamental UV theories, such as unified theories with gravity, which would describe our universe. Once we consider such a nonrenormalizable theory, higher-derivative interactions appear which are absent in a renormalizable system. For example, the effective field theory of superstring theory contains such derivative interactions as well as non-derivative ones. However, we may need to be careful of higher-derivative interactions. As is well known, derivative interactions beyond second order can lead to a problem called the Ostrogradsky instability [1, 2], which causes a Hamiltonian unbounded from below. However, even when an interaction term contains more than two derivatives, some specific classes of such interactions do not exhibit the instability. One sufficient condition is that the equations of motion (E.O.M) in the system are at most second order differential equations. In a scalartensor system, the Horndeski class action is one example of a ghost-free higher-derivative system [3, 4]. Recently, the higher-derivative action of a Proca field is also drawing attention in the context of cosmology [5-7]. Although the instability itself is not a real problem if there is a ghost-free UV completion, the investigation of ghost-free higher-derivative interactions would be an interesting problem in its own right.

Supersymmetry (SUSY) was proposed to solve the hierarchy problem of the standard model and is one of important tools to investigate physics beyond the standard model. The effective theories of UV complete SUSY theories such as superstring theory naturally contain higher-derivative terms as corrections to the leading two-derivative terms. However, higher-derivative terms in SUSY field theories often encounters the so-called auxiliary field problem [8-12]: the action contains spacetime derivatives of the SUSY auxiliary fields 
( $F$ and $\boldsymbol{D}$ for chiral and vector multiplets, respectively), so that one cannot eliminate them by their E.O.M. It was recognized [8-12] that this problem may occur in several higher-derivative chiral models such as a Wess-Zumino term [13, 14], and Skyrme [15] and Faddeev-Skyrme [16] models. In most cases, the derivatives on the auxiliary fields and the higher-derivative ghosts come up together [17]. ${ }^{1}$ Finally, a systematic classification of higher-derivative terms which are free from ghosts as well as the auxiliary field problem was given in refs. [19-22]. Such terms were used in several SUSY higher-derivative chiral models; low-energy effective theory [23-26], coupling to supergravity (SUGRA) [21,27] and its applications [20] to Galileons [28] and ghost condensation [22], a Dirac-Born-Infeld (DBI) inflation [29], flattening of the inflaton potential [30,31], topological solitons such as a BPS baby Skyrme model [32-37], a Skyrme model [38-40], BPS solitons [34, 35, 41] and their effective action [42], nonlinear realizations [43], and a possibility of modulated vacua [44, 45]. In addition, different ghost-free higher-derivative actions of a chiral superfield are possible in the global [46] and SUGRA [47] cases as well as a non-local theory [48].

On the other hand, there have been no such systematic studies for vector multiplets so far. There are only few examples of ghost-free actions of a vector multiplet. One is the DBI action in $4 \mathrm{D} \mathcal{N}=1$ SUSY [49-51]. Since it is totally written in terms of the field strength $F_{m n}=\partial_{m} A_{n}-\partial_{n} A_{m}$ without any additional derivative operators, its E.O.M is of a second order, and no additional (ghost) mode appears. The coupling to SUGRA has also been discussed in refs. [49, 52-54]. The SUSY Euler-Heisenberg action is one of ghost-free extensions of the Maxwell system [27, 55-57]. The others are a higher derivative extension of a scalar potential in SUGRA [55] and a SUSY extension of non-linear self-dual actions for vector gauge fields $[52,53,58-60]$.

In this paper, we systematically study ghost-free higher-derivative actions of vector multiplets in $4 \mathrm{D} \mathcal{N}=1$ SUSY theories. The known example of the DBI action gives us useful insights to find possible ghost-free interactions. To construct the most general ghost-free SUSY higher-derivative actions, the nontrivial question is the conditions for the absence of the higher-derivative ghosts. We will follow the following simple argument as a guiding principle: if the bosonic part of the action is ghost-free, the total system would be ghost-free as well. This argument would be justified unless some fermions in the system condensate and acquire vacuum expectation values. ${ }^{2}$ Another nontrivial point is the auxiliary field problem. Typically, auxiliary fields become dynamical due to higherderivative interactions, as mentioned above. In most known cases, the dynamical auxiliary field leads to a ghost (super)field. We will show that the ghost-free higher-derivative action does not have any propagating auxiliary field but has higher powers of the auxiliary field, yielding corrections to the D-term potential. We will show an example of such a corrected D-term potential, which has nontrivial vacua due to the corrections. We also give a matter coupling to higher-derivative vector multiplets.

\footnotetext{
${ }^{1}$ If one can eliminate a ghost with a gauging by introducing an auxiliary vector superfield, one can construct a model with a propagating "auxiliary" field without a ghost [18], which we may call a ghostbuster mechanism.

${ }^{2}$ This discussion is applied for bosonic ghosts and does not exclude a possibility of fermionic ghosts. For instance, a fermionic ghost exists when the vacuum energy becomes negative [44, 45]. For a general discussion for fermionic ghosts, see refs. [61, 62].
} 
This paper is organized as follows. In section 2, we show a nontrivial example which has a higher-derivative ghost for comparison. Then we move to the construction of ghostfree models in section 3. First, we discuss possible building blocks for ghost-free higherderivative actions in section 3.1. With such ingredients, we find the general SUSY action whose bosonic part is free from ghosts. We also show the corresponding on-shell action. In section 3.2, we show some concrete examples of ghost-free models, their bosonic actions, and the vacua realized by corrected D-term potentials. We first show that the well-known SUSY DBI action is obtained as a particular example of our construction. We next propose a simple example. In the example, there are two kinds of solutions of the E.O.M for the auxiliary D-term, which give so-called canonical and non-canonical branches. On the one hand, in the canonical branch, the bosonic action consists of the standard quadratic kinetic term of the field strength (Maxwell term) and a higher-derivative term of fourth order. On the other hand, in the non-canonical branch, the action contains only a quartic higherderivative term of the field strength without the Maxwell term [27, 56]. In addition, we mention possible couplings to matter fields in section 4 . We conclude in section 5 with a brief discussion. In appendix A, the convention of a vector superfield is summarized. Throughout this paper, we will use the convention of Wess and Bagger [63].

\section{A higher-derivative action for a vector multiplet with a ghost}

Before going to ghost-free models, we show a nontrivial example of a higher-derivative action of a vector multiplet $V$ with a ghost mode for comparison. The convention of the vector multiplet is summarized in appendix $\mathrm{A}$.

We consider the following system,

$$
\mathcal{L}=\left(\int d^{2} \theta \frac{1}{4} W^{\alpha} W_{\alpha}+\text { h.c. }\right)+\int d^{4} \theta f\left(D^{\alpha} W_{\alpha}\right) .
$$

Here, $W_{\alpha}=-\frac{1}{4} \bar{D}^{2} D_{\alpha} V$ is a gauge invariant chiral superfield, and $f$ is a real function such that its first derivative $f^{\prime}$ is a non-constant function. ${ }^{3}$ To show the existence of a ghost, we rewrite the action as follows, Since $\bar{D}^{2}\left(D^{\alpha} W_{\alpha}\right)=D^{2}\left(D^{\alpha} W_{\alpha}\right)=0, D^{\alpha} W_{\alpha}$ is a real linear superfield and hence we can rewrite the action as

$$
\mathcal{L}=\left(\int d^{2} \theta \frac{1}{4} W^{\alpha} W_{\alpha}+\text { h.c. }\right)+\int d^{4} \theta f(L)+\int d^{4} \theta U\left(L-D^{\alpha} W_{\alpha}\right),
$$

where $L$ and $U$ are a real linear and a real superfield, respectively. Note that the real superfield $U$ is an additional vector superfield, and this action is invariant under the following gauge transformation for $U$,

$$
U \rightarrow U-\Sigma^{\prime}-\bar{\Sigma}^{\prime}
$$

where $\Sigma^{\prime}$ is a chiral superfield. The variation of $U$ gives the constraint $L=D^{\alpha} W_{\alpha}$, which reproduces the original action. If we instead perform a super-partial integral, we obtain

$$
\mathcal{L}=\int d^{2} \theta\left(\frac{1}{4} W^{\alpha} W_{\alpha}+\frac{1}{2} \widehat{W}^{\alpha} W_{\alpha}+\text { h.c. }\right)+\int d^{4} \theta[f(L)+U L],
$$

\footnotetext{
${ }^{3}$ If $f$ is a constant or a linear function, the last term in the Lagrangian vanishes.
} 
where we have used

$$
\int d^{4} \theta U D^{\alpha} W_{\alpha}=-\int d^{4} \theta D^{\alpha} U W_{\alpha}=-\int d^{2} \theta \widehat{W}^{\alpha} W_{a},
$$

with $\widehat{W}_{\alpha}=-\frac{1}{4} \bar{D}^{2} D_{\alpha} U$. By diagonalizing the kinetic terms of $W^{\alpha}$ and $\widehat{W}^{\alpha}$ in eq. (2.4), we find that one combination of the vector superfields has a kinetic term with a wrong sign implying the existence of a ghost vector superfield. Note that our action in eq. (2.1) is a generalization of an action with a ghost given in ref. [57].

We may trade the linear superfield for a chiral superfield, which makes the situation clearer. The part of the action containing the linear superfield can be rewritten as

$$
\int d^{4} \theta f(\tilde{L})+\int d^{4} \theta U \tilde{L}+\int d^{4} \theta \tilde{L}(\Phi+\bar{\Phi}),
$$

where $\tilde{L}$ is a general superfield and $\Phi$ is a chiral superfield. The variation of $\Phi$ gives the constraint for a linear superfield $\bar{D}^{2} \tilde{L}=0$, which reproduces $\tilde{L}=L$, whereas the variation of $\tilde{L}$ gives $f^{\prime}(\tilde{L})+(\Phi+\bar{\Phi}+U)=0$. As long as this equation can be solved (implicitly) as $\tilde{L}=\tilde{L}(\Phi+\bar{\Phi}+U)$, the action can be rewritten as

$$
\mathcal{L}=\int d^{2} \theta\left(\frac{1}{4} W^{\alpha} W_{\alpha}+\frac{1}{2} \widehat{W}^{\alpha} W_{\alpha}+\text { h.c. }\right)+\int d^{4} \theta g(\Phi+\bar{\Phi}+U),
$$

where

$$
g(\Phi+\bar{\Phi}+U)=\left.\{f(\tilde{L})+\tilde{L}(\Phi+\bar{\Phi}+U)\}\right|_{\tilde{L}=\tilde{L}(\Phi+\bar{\Phi}+U)} .
$$

Note that $\Phi$ is a Stückelberg superfield which transforms as $\Phi \rightarrow \Phi+\Sigma^{\prime}$ under the gauge transformation of $U$ in eq. (2.3).

Thus we have shown that the higher-derivative interaction in eq. (2.1) leads to a ghost vector superfield. Note that if $f^{\prime}$ is a constant, the equation $f^{\prime}(\tilde{L})+(\Phi+\bar{\Phi}+U)=0$ can be solved with respect to $U$, which then becomes a composite superfield. Consequently, no ghost fields arise.

\section{Ghost-free higher-derivative actions for vector multiplet}

In this section, we discuss possible ghost-free higher-derivative actions of a vector multiplet. Since a massive vector superfield belongs to a reducible representation, we focus only on a massless gauge superfield. First, we consider building blocks for a higher-derivative action. To preserve the gauge symmetry, we need to use the gauge invariant superfield $W_{\alpha}=-\frac{1}{4} \bar{D}^{2} D_{\alpha} V$, which we call the "gaugino superfield". According to the Ostrogradsky's method, ghost modes appear if the equation of motion is higher than second-order differential equations. Since the $\theta$-component of $W_{\alpha}$ contains the field strength $F_{m n}$, the spacetime derivatives of $W_{\alpha}$ in the superfield action would lead to higher-derivative terms in the E.O.M, and consequently give rise to ghost modes. For example, a simple interaction with spacetime derivatives $\int d^{2} \theta \partial^{m} W^{\alpha} \partial_{m} W_{\alpha}$ leads to a fourth-order differential equation for the gauge field $A_{m}$. 


\subsection{The building blocks of ghost-free higher-derivative terms}

In this subsection, we write down possible building blocks of higher-derivative actions. As we mentioned in section 1, we only focus on purely bosonic parts of higher-derivative actions and require them to be ghost-free. We propose the following three simple conditions to realize ghost-free bosonic terms:

- To obtain a manifestly gauge invariant action, we require that the integrand of the superfield action is written in terms of the gaugino superfield $W_{\alpha}$ rather than the vector superfield $V$. Consequently, the bosonic part of the action is written totally in terms of the field strength, which is manifestly gauge invariant.

- The Lagrangian has no spacetime derivatives of the field strengths such as $\partial_{m} F_{n p}$, which may lead to ghosts. ${ }^{4}$

- SUSY invariants which we focus on should have at least one purely bosonic term. Only such terms are relevant for the presence/ absence of ghosts.

Taking into account these criteria, we find the following most general form of the higher-derivative Lagrangians:

$$
\mathcal{L}=\left(\int d^{2} \theta \mathcal{F}(H) W^{2}+\text { h.c. }\right)+\int d^{4} \theta \mathcal{G}(H, \bar{H}, \widehat{\boldsymbol{D}}) W^{2} \bar{W}^{2},
$$

where (anti-)chiral superfield $H(\bar{H})$ and real superfield $\widehat{\boldsymbol{D}}$ are defined by

$$
H \equiv-\frac{1}{4} \bar{D}^{2} \bar{W}^{2}, \quad \bar{H} \equiv-\frac{1}{4} D^{2} W^{2}, \quad \widehat{\boldsymbol{D}} \equiv-\frac{1}{2} D^{\alpha} W_{\alpha}=-\frac{1}{2} \bar{D}_{\dot{\alpha}} \bar{W}^{\dot{\alpha}} .
$$

Here $\mathcal{F}$ and $\mathcal{G}$ are arbitrary functions of arguments, which are holomorphic and real scalar superfields, respectively. In the following, we explain how the Lagrangian in eq. (3.1) can be obtained from the above three conditions.

Firstly, we focus on the "irreducible" components of the gaugino superfield, which is not made of other (lower) components of a supermultiplet. In the gaugino superfield, there is only one purely bosonic irreducible component ${ }^{5}$

$$
D_{\alpha} W_{\beta} \mid=-i\left(\sigma^{m n} \epsilon\right)_{\alpha \beta} F_{m n}-\epsilon_{\alpha \beta} \boldsymbol{D},
$$

where the vertical bar denotes $\theta=\bar{\theta}=0$ projection on a superfield, and $\left(\sigma^{m n} \epsilon\right)_{\alpha \beta}=$ $\frac{1}{4}\left(\sigma_{\alpha \dot{\gamma}}^{m} \bar{\sigma}^{n \dot{\gamma} \delta}-\sigma_{\alpha \dot{\gamma}}^{n} \bar{\sigma}^{m \dot{\gamma} \delta}\right) \epsilon_{\delta \beta}$. This implies that the numbers of derivatives $D_{\alpha}$ and $\bar{D}^{\dot{\alpha}}$ in the component action should be equal to those of the gaugino superfield $W_{\alpha}$ and $\bar{W}^{\dot{\alpha}}$, respectively. In other words, the bosonic part $\mathcal{L}_{b}$ of the Lagrangian should take the form of

$$
\mathcal{L}_{b}=\mathcal{L}_{b}\left(D_{\alpha} W_{\beta}\left|, \bar{D}^{\dot{\alpha}} \bar{W}^{\dot{\beta}}\right|\right) .
$$

\footnotetext{
${ }^{4}$ Here, we do not mean the absence of the terms e.g. $A^{m} \partial^{n} F_{m n}$. This conflicts with the first requirement that the Lagrangian is a function of field strength.

${ }^{5}$ Note that $F_{m n}$ is not an irreducible component in the vector superfield $V$. However, we can regard $F_{m n}$ as an irreducible component in the gaugino superfield $W_{\alpha}$.
} 
Furthermore, the Lorentz invariance requires the Lagrangian $\mathcal{L}_{b}$ to be composed of Lorentz invariant combinations of $D_{\alpha} W_{\beta} \mid$ and $\bar{D}^{\dot{\alpha}} \bar{W}^{\dot{\beta}} \mid$. We can show that the following three Lorentz invariant combinations are independent building blocks of the Lagrangian

$$
D^{\alpha} W_{\alpha}, \quad D^{\alpha} W^{\beta} D_{\alpha} W_{\beta}, \quad \bar{D}_{\dot{\alpha}} \bar{W}_{\dot{\beta}} \bar{D}^{\dot{\alpha}} \bar{W}^{\dot{\beta}}
$$

This follows from the irreducible decomposition of $D_{\alpha} W_{\beta}$

$$
D_{\alpha} W_{\beta}=\frac{1}{2} \epsilon_{\alpha \beta} D^{\gamma} W_{\gamma}+D_{(\alpha} W_{\beta)}
$$

and the relation

$$
D^{(\alpha} W^{\beta)} D_{(\beta} W_{\gamma)}=\delta_{\gamma}^{\alpha}\left(\frac{1}{2} D^{\beta} W^{\delta} D_{\beta} W_{\delta}-\frac{1}{4}\left(D^{\beta} W_{\beta}\right)^{2}\right) .
$$

Here, the parenthesis for spinors means the symmetrization: $D_{(\alpha} W_{\beta)}=\frac{1}{2}\left(D_{\alpha} W_{\beta}+D_{\beta} W_{\alpha}\right)$. Similar equations hold for $\bar{D}^{\dot{\alpha}} \bar{W}^{\dot{\beta}}$. Thus we can express Lorentz invariants given by contractions of the spinors of $D_{\alpha} W_{\beta}$ and $\bar{D}^{\dot{\alpha}} \bar{W}^{\dot{\beta}}$ in terms of (3.5). ${ }^{6}$ Note that $D^{\alpha} W_{\alpha}=-2 \widehat{\boldsymbol{D}}$ is a real superfield. We can replace $D^{\alpha} W^{\beta} D_{\alpha} W_{\beta}$ with $\bar{H}$ since

$$
D^{\alpha} W^{\beta} D_{\alpha} W_{\beta}=2 \bar{H}+\left(D^{2} W_{\alpha}\right) W^{\alpha},
$$

and the last term has only fermionic terms, which are irrelevant to our discussion. Therefore, the Lagrangian should take the form

$$
\mathcal{L}_{b}=\mathcal{L}_{b}(\bar{J} \equiv H|, J \equiv \bar{H}|, \boldsymbol{D}=\widehat{\boldsymbol{D}} \mid),
$$

where

$$
J:=-\frac{1}{4} D^{2} W^{2} \mid=-\frac{1}{2} F^{m n} F_{m n}+\frac{i}{2} F_{m n} \tilde{F}^{m n}+D^{2}-2 i \lambda^{\alpha}\left(\bar{\sigma}^{m}\right)_{\alpha \dot{\beta}} \partial_{m} \bar{\lambda}^{\dot{\beta}}
$$

and $\tilde{F}^{m n}=\frac{1}{2} \epsilon^{m n p q} F_{p q}$. The form (3.9) simply implies that the Lagrangian should be a function of $\left(F_{m n} F^{m n}, F_{m n} \tilde{F}^{m n}, \boldsymbol{D}\right)$.

In order to embed the bosonic expression (3.9) to a SUSY system, one needs to describe it as a superspace integration of superfields. Since $\int d^{2} \theta$ and $\int d^{4} \theta$ integrations are equivalent to acting two and four spinor derivatives on superfields, the super-integrands have to be respectively proportional to $W_{\alpha} W_{\beta}$ for $\int d^{2} \theta$ integrals and $W_{\alpha} W_{\beta} \bar{W}_{\dot{\alpha}} \bar{W}_{\dot{\beta}}$ for $\int d^{4} \theta$ integrals so that the resulting component action takes the form $\mathcal{L}(H, \bar{H}, \boldsymbol{D})$. From these observations, we obtain the most general Lagrangian satisfying all the conditions: ${ }^{7}$

$$
\mathcal{L}=\left(\int d^{2} \theta \mathcal{F}(H) W^{2}+\text { h.c. }\right)+\int d^{4} \theta \mathcal{G}(H, \bar{H}, \widehat{\boldsymbol{D}}) W^{2} \bar{W}^{2},
$$

where $\mathcal{F}$ and $\mathcal{G}$ are arbitrary holomorphic and real scalar functions, respectively.

\footnotetext{
${ }^{6}$ Note that there are ambiguities of choosing the independent quantities. For example, we can choose $D^{\alpha} W^{\beta} D_{\beta} W_{\alpha}$ instead of $D^{\alpha} W^{\beta} D_{\alpha} W_{\beta}$. However, the number of the independent quantities does not change.

${ }^{7}$ Note that $W_{\alpha} W_{\beta}$ can be rewritten as $W_{\alpha} W_{\beta}=\frac{1}{2} \epsilon_{\alpha \beta} W^{2}$.
} 
We note that $\widehat{\boldsymbol{D}} \propto D^{\alpha} W_{\alpha}$ dependence causes no ghost mode in contrast to the previous section since $\mathcal{G} W^{2} \bar{W}^{2}$ is not invertible with respect to $D^{\alpha} W_{\alpha}$. Then, repeating the procedure discussed in the previous section, we find out that the vector superfield $U$ satisfying $\mathcal{G}^{\prime} W^{2} \bar{W}^{2}+\Phi+\bar{\Phi}+U=0$ is always a composite superfield. Therefore, the function $\mathcal{G}$ is allowed to be a function of $\widehat{\boldsymbol{D}}$.

Among $H, \bar{H}$, and $\widehat{\boldsymbol{D}}$, only $H$ is a chiral superfield, and hence the chiral superfield $\mathcal{F}$ needs to be a holomorphic function of $H$. As long as $\mathcal{F}$ is a smooth function around $H=0$, it can always be written as

$$
\mathcal{F}(H)=\tau+h(H) H
$$

where $\tau$ is a constant and $h(H)$ is a holomorphic function. The second term can be absorbed into $\mathcal{G}$ due to the identity

$$
\int d^{2} \theta h(H) H W^{2}=-\frac{1}{4} \int d^{2} \theta \bar{D}^{2}\left(h(H) \bar{W}^{2} W^{2}\right)=\int d^{4} \theta h(H) W^{2} \bar{W}^{2} .
$$

Therefore we can set $\mathcal{F}=\tau=$ const. Consequently, the bosonic part of Lagrangian takes the form

$$
\mathcal{L}_{b}=(\tau J+\text { h.c. })+\mathcal{G}(\bar{J}, J, \boldsymbol{D})|J|^{2} .
$$

The first term gives the standard quadratic terms (Maxwell, theta and D-terms), while the second term is the higher-derivative correction, which is required to be proportional to $|J|^{2}$ due to SUSY. ${ }^{8}$

Finally, we note that there is no derivative acting on the auxiliary field $\boldsymbol{D}$ in our general action, and hence the solution of its E.O.M can be expressed as a local function of physical fields, i.e. $F_{m n}$ without derivatives on it. ${ }^{9}$ Therefore, if we assign an appropriate sign for the quadratic term, the on-shell action is always ghost-free as we expected.

\subsection{Examples of ghost-free higher-derivative action for vector multiplet}

In the previous subsection, we have derived the general SUSY action, which has a ghost-free purely bosonic part. In this section, we consider some specific examples. We can construct various models by choosing $\mathcal{F}$ and $\mathcal{G}$ in our general action (3.1).

First, let us choose $\mathcal{F}=1 / 4 g^{2}$ and $\mathcal{G}=\mathcal{G}\left(D^{\alpha} W_{\alpha}\right)$ :

$$
\mathcal{L}=\left(\frac{1}{4 g^{2}} \int d^{2} \theta W^{2}+\text { h.c. }\right)+\int d^{4} \theta \mathcal{G}\left(D^{\alpha} W_{\alpha}\right) W^{2} \bar{W}^{2} .
$$

Here, $g$ is a coupling constant. Note that the reality of $\mathcal{G}$ is satisfied because $D^{\alpha} W_{\alpha}$ is a real superfield $D^{\alpha} W_{\alpha}=\bar{D}_{\dot{\alpha}} \bar{W}^{\dot{\alpha}}$. Since $D^{\alpha} W_{\alpha}=-2 \boldsymbol{D}$, to the bosonic part, $\mathcal{G}$ only gives higher-order terms of auxiliary fields, i.e.

$$
\mathcal{L}_{b}=\frac{1}{4 g^{2}}(J+\bar{J})+\mathcal{G}(-2 \boldsymbol{D})|J|^{2} .
$$

\footnotetext{
${ }^{8}$ Thanks to this property, the self-dual (instanton) solution of the Yang-Mills equation would not be modified by the additional higher-derivative terms in the non-Abelian extension of our model.

${ }^{9}$ In the presence of matter fields, $\boldsymbol{D}$ can also depend on matter fields without derivatives.
} 
Second, for $\mathcal{G}=\mathcal{G}(H, \bar{H})$, the corresponding action is given by

$$
\mathcal{L}=\left(\frac{1}{4 g^{2}} \int d^{2} \theta W^{2}+\text { h.c. }\right)+\int d^{4} \theta \mathcal{G}(H, \bar{H}) W^{2} \bar{W}^{2} .
$$

In this case, the bosonic action is

$$
\mathcal{L}_{b}=\frac{1}{4 g^{2}}(J+\bar{J})+\mathcal{G}(\bar{J}, J)|J|^{2} .
$$

Contrary to the previous example, this action contains higher order terms of the field strength $F_{m n}$. This difference would be useful for model buildings.

Obviously, these action leads to a second order E.O.M for the vector field. Furthermore, there is no derivative interaction of the auxiliary field $\boldsymbol{D}$ as we mentioned. Hence, the system does not have any additional degrees of freedom. Since the number of bosonic degrees of freedom does not change, that of fermions also remains the same due to SUSY.

The DBI action is a well-known example of a ghost-free higher-derivative action. It is realized with the following set of choices [49, 50],

$$
\begin{aligned}
& \mathcal{F}=\frac{1}{4 g^{2}}=\frac{\mu \alpha^{2}}{4}, \quad \text { with } \alpha \equiv \frac{1}{\sqrt{\mu g^{2}}} \\
& \mathcal{G}=\frac{\mu \alpha^{4}}{4}\left(1-\frac{\alpha^{2}}{4}(H+\bar{H})+\sqrt{1-\frac{\alpha^{2}}{2}(H+\bar{H})+\frac{\alpha^{4}}{16}(H-\bar{H})^{2}}\right)^{-1} .
\end{aligned}
$$

The corresponding bosonic Lagrangian is given by

$$
\begin{aligned}
\mathcal{L}_{b} & =\frac{\mu \alpha^{2}}{4}(J+\bar{J})+\frac{\mu \alpha^{4}}{4}\left(1-\frac{\alpha^{2}}{4}(J+\bar{J})+\sqrt{1-\frac{\alpha^{2}}{2}(J+\bar{J})+\frac{\alpha^{4}}{16}(J-\bar{J})^{2}}\right)^{-1}|J|^{2} \\
& =\mu-\mu \sqrt{1-\frac{\alpha^{2}}{2}(J+\bar{J})+\frac{\alpha^{4}}{16}(J-\bar{J})^{2}} \\
& =\mu-\mu \sqrt{1+\frac{\alpha^{2}}{2} F_{m n} F^{m n}-\alpha^{2} D^{2}-\frac{\alpha^{4}}{16}\left(\tilde{F}^{m n} F_{m n}\right)^{2}} \\
& =\mu-\mu \sqrt{-\operatorname{det}\left(\eta_{m n}+\alpha F_{m n}\right)-\alpha^{2} \boldsymbol{D}^{2}}
\end{aligned}
$$

We find that $\boldsymbol{D}=0$ is the unique solution of E.O.M for $\boldsymbol{D}$. Thus we obtain the on-shell action

$$
\int d^{4} x \mu\left(-\sqrt{-\operatorname{det}\left(\eta_{m n}+\alpha F_{m n}\right)}+1\right)
$$

Next, let us consider the following simplest example [27, 55-57]:

$$
S=\frac{1}{4} \int d^{4} x d^{2} \theta\left(\frac{1}{g^{2}}+\frac{1}{4 \Lambda} \bar{D}^{2} \bar{W}^{2}\right) W^{2}+\text { h.c. }
$$

Here, $\Lambda$ is a real parameter of mass dimension four. This action is realized by choosing $\mathcal{F}$ as

$$
\mathcal{F}=\frac{1}{4 g^{2}}+\frac{1}{16 \Lambda} \bar{D}^{2} \bar{W}^{2}
$$


The bosonic part of the action $S_{b}$ can be calculated as

$S_{b}=\int d^{4} x\left[-\frac{1}{4 g^{2}} F^{m n} F_{m n}+\frac{1}{2 g^{2}} \boldsymbol{D}^{2}-\frac{1}{2 \Lambda}\left(-\frac{1}{2} F^{m n} F_{m n}+\boldsymbol{D}^{2}\right)^{2}-\frac{1}{2 \Lambda}\left(\frac{1}{2} F^{m n} \tilde{F}_{m n}\right)^{2}\right]$.

The E.O.M for the auxiliary field $\boldsymbol{D}$ reads:

$$
0=\boldsymbol{D}\left[\frac{1}{g^{2}}+\frac{2}{\Lambda}\left(\frac{1}{2} F^{m n} F_{m n}-\boldsymbol{D}^{2}\right)\right] .
$$

Thus, we have the following three solutions for the auxiliary field:

$$
\boldsymbol{D}=0, \quad \pm \sqrt{\frac{1}{2}\left(F^{m n} F_{m n}+\frac{\Lambda}{g^{2}}\right)} .
$$

The on-shell action in the corresponding branches can be written as follows.

- The canonical branch (the case of $\boldsymbol{D}=0$ ):

$$
S_{b}=\int d^{4} x\left[-\frac{1}{4 g^{2}} F^{m n} F_{m n}-\frac{1}{8 \Lambda}\left(F^{m n} F_{m n}\right)^{2}-\frac{1}{8 \Lambda}\left(F^{m n} \tilde{F}_{m n}\right)^{2}\right] .
$$

This action contains the higher-derivative terms $\left(F^{m n} F_{m n}\right)^{2}$ and $\left(F^{m n} \tilde{F}_{m n}\right)^{2}$ as well as the standard quadratic term $F^{m n} F_{m n}$. In this case, the higher-order terms vanish in the limit $|\Lambda| \rightarrow \infty$.

- The non-canonical branch (the case of $\boldsymbol{D}= \pm \sqrt{\left(F^{m n} F_{m n}+\Lambda / g^{2}\right) / 2}$ ):

$$
S=\int d^{4} x\left[-\frac{1}{8 \Lambda}\left(F^{m n} \tilde{F}_{m n}\right)^{2}+\frac{\Lambda}{8 g^{4}}\right] .
$$

An interesting property of this action is the absence of the quadratic kinetic term. Therefore, we call this branch the non-canonical one. Since $\boldsymbol{D}$ is real, $F^{m n} F_{m n}+\Lambda / g^{2}$ should be non-negative. Therefore the solution of non-canonical branch is consistent only when

$$
F^{m n} F_{m n}+\frac{\Lambda}{g^{2}} \geq 0
$$

This inequality shows that $F_{m n}=0$ can be a consistent solution of the E.O.M only when the parameter $\Lambda$ is positive. ${ }^{10}$ Note that this branch becomes ill-defined in the limit $|\Lambda| \rightarrow \infty$.

As shown above, there exist multiple branches due to the higher-order terms of the auxiliary field $\boldsymbol{D}$. The presence of the multiple branches has been known in the case of chiral multiplets $\Phi$ with the action $\sim \int d^{4} \theta\left|D^{\alpha} \Phi D_{\alpha} \Phi\right|^{2}$, where the SUSY higher-derivative term involves a quartic interaction of the F-term [32-35]. Similarly to the present case, the F-term has three solutions, and they lead to a canonical branch with the standard kinetic term and non-canonical branches without a second-order derivative term.

\footnotetext{
${ }^{10}$ The case in which $\Lambda$ is negative is investigated in ref. [56]. In such a case, $F^{m n} F_{m n}$ cannot become zero from the condition in eq. (3.29).
} 


\section{A coupling to matter fields}

So far, we have discussed higher-derivative actions composed purely of vector superfields. To discuss more general and phenomenological models, we consider the following higherderivative system coupled to the matter chiral superfields $\Phi_{1}$ and $\Phi_{2}$ :

$$
\begin{aligned}
\mathcal{L}= & \frac{1}{2} \int d^{4} \theta\left(\bar{\Phi}_{1} e^{V} \Phi_{1}+\bar{\Phi}_{2} e^{-V} \Phi_{2}+\mathcal{G}\left(H, \bar{H}, \widehat{\boldsymbol{D}}, \Phi_{1}, \bar{\Phi}_{1} e^{V}, \Phi_{2}, \bar{\Phi}_{2} e^{-V}\right) W^{2} \bar{W}^{2}+\xi V\right) \\
& +\frac{1}{4 g^{2}} \int d^{2} \theta W^{2}+\text { h.c. }
\end{aligned}
$$

where $\xi$ is a real parameter called the Feyet-Iliopoulos (FI) parameter. The bosonic part of the Lagrangian is given by

$$
\begin{aligned}
\mathcal{L}_{b}= & -\mathcal{D}^{m} A_{1} \mathcal{D}_{m} A_{1}^{*}-\mathcal{D}^{m} A_{2} \mathcal{D}_{m} A_{2}^{*}+A_{1} A_{1}^{*} \boldsymbol{D}-A_{2} A_{2}^{*} \boldsymbol{D}+F_{1} F_{1}^{*}+F_{2} F_{2}^{*}+\xi \boldsymbol{D} \\
& -\frac{1}{4 g^{2}} F^{m n} F_{m n}+\frac{1}{2 g^{2}} \boldsymbol{D}^{2}+\mathcal{G}\left(\bar{J}, J, \boldsymbol{D}, A_{i}, A_{i}^{*}\right)|J|^{2},
\end{aligned}
$$

where $A_{i}=\Phi_{i} \mid(i=1,2)$ are complex scalar fields, and $F_{i}=-\frac{1}{4} D^{2} \Phi_{i}$ are auxiliary fields for the chiral superfields, and $\mathcal{D}_{m}$ is a gauge covariant vector derivative. Here, We find that there are no derivatives acting on the auxiliary field $\boldsymbol{D}$ as well as the auxiliary fields $F_{i}$ for the chiral superfields $\Phi_{i}$. Therefore, the solutions to the E.O.M for $\boldsymbol{D}$ and $F_{i}$ are functions of $A_{i}, A_{i}^{*}$ and $F_{m n}$ without spacetime derivatives acting on them. Thus, there are no ghost modes even when the vector superfield is coupled to matter fields.

\section{Summary and discussion}

We have investigated ghost-free higher-derivative actions of a vector multiplet in $4 \mathrm{D} \mathcal{N}=1$ SUSY models. As we have shown in section 2, a naive higher-derivative extension leads to a ghost mode, even though it preserves the gauge symmetry. We have taken rather general conditions that lead to the ghost-free bosonic action: the bosonic part of the Lagrangian must be a function of $F_{m n}$ and $\boldsymbol{D}$ without derivatives acting on them. In addition, it should be expressed by SUSY invariants. These simple requirements lead to the general action given in eq. (3.1), which is characterized by a holomorphic function $\mathcal{F}=\mathcal{F}\left(\bar{D}^{2} \bar{W}^{2}\right)$ and a real function $\mathcal{G}$ of $D^{\alpha} W_{\alpha}, \bar{D}^{2} \bar{W}^{2}$ and $D^{2} W^{2}$. We have also shown some examples with specific $\mathcal{F}$ and $\mathcal{G}$. As we mentioned, the SUSY DBI action is one of particular known examples in our framework. In general, higher-derivative terms yield higher powers of the auxiliary field $\boldsymbol{D}$, and consequently the E.O.M for $\boldsymbol{D}$ admits multiple solutions. In the other example in eq. (3.22), the E.O.M for $\boldsymbol{D}$ gives two types of solutions: the canonical branch, in which the bosonic action consists of the standard quadratic kinetic term of the field strength (the Maxwell term) and a fourth order higher-derivative term, and the non-canonical branch, which is composed only of a fourth order higher-derivative term without the standard kinetic term. We have also shown that the matter coupled case discussed in section 4 still satisfies our criteria for the absence of ghosts. The resultant action would have a deformed D-term potential, which has potentially interesting applications to model buildings. 
There are many possible applications of our results. Since it is straightforward to extend the action (3.1) to SUGRA, we can consider the inflationary model buildings. Indeed, the DBI extension of a matter-coupled vector superfield action leads to a scalar potential flattened by higher-order terms [64]. It would be interesting to consider the SUSY breaking due to the higher-dimensional operators $[56,65,66]$. It may also be possible to apply our framework to construct effective 4D models of superstring, where the higher-order D-term plays an important role, since the higher-order F-term contribution has been applied to effective string models of moduli stabilization [67] as well as inflation [68].

Since a non-canonical branch of the E.O.M for the auxiliary field in higher-derivative chiral models gives rise to BPS equations for BPS baby Skyrmions [32-35], the noncanonical branch of our vector superfield in eq. (3.28) may give a new kind of BPS topological solitons or instantons. It is also interesting to look for new BPS solitons in the matter coupled system discussed in section 4 .

The generalization to a non-Abelian case is straightforward but interesting for various applications, e.g., in inflation [69-71]. In such a case, the higher-order action should be further restricted by the non-Abelian symmetry. In addition, our systematic analysis would be applicable to the construction of a ghost-free action of a linear superfield, which was studied in a few cases $[51,72,73]$. As a generalization, we can also consider the combination of the ghost-free higher-derivative actions of matter fields and higher curvature in SUGRA. It would also be interesting to see whether compositions of ghost-free models remain ghostfree or not. We will address these issues elsewhere. Finally, in certain chiral models, a ghost chiral superfield can be gauged out by introducing an auxiliary vector superfield without a kinetic term [18]. It is an open question whether the ghost in a vector superfield discussed in section 2 can be eliminated by introducing an auxiliary two-form tensor gauge field allowing a gauge transformation of a two-form tensor.

\section{Acknowledgments}

This work is supported by the Ministry of Education, Culture, Sports, Science (MEXT)Supported Program for the Strategic Research Foundation at Private Universities "Topological Science" (Grant No. S1511006). The work of M. N. is also supported in part by a Grant-in-Aid for Scientific Research on Innovative Areas "Topological Materials Science" (KAKENHI Grant No. 15H05855) from the MEXT of Japan, and by the Japan Society for the Promotion of Science (JSPS) Grant-in-Aid for Scientific Research (KAKENHI Grant No. 16H03984). The work of R. Y. is supported by Research Fellowships of JSPS for Young Scientists Grant Number 16J03226. Y. Y. is supported by SITP and by the NSF Grant PHY-1720397.

\section{A Convention for vector superfield}

In this appendix, we summarize our convention for vector superfields. The convention is the same as ref. [63]. 
We denote a real vector superfield as $V$. The gauge field $A_{m}$ is embedded into the vector component of $V$ as

$$
A_{m}=-\frac{1}{4} \bar{\sigma}_{m}^{\dot{\alpha} \alpha}\left[D_{\alpha}, \bar{D}_{\dot{\alpha}}\right] V
$$

The vector superfield $V$ transforms under a $\mathrm{U}(1)$ gauge transformation as

$$
V \rightarrow V+\Sigma+\bar{\Sigma}
$$

where $\Sigma$ is a chiral superfield $\bar{D}_{\dot{\alpha}} \Sigma=0$. The gaugino superfield is defined by

$$
W_{\alpha}=-\frac{1}{4} \bar{D}^{2} D_{\alpha} V
$$

which is invariant under the $\mathrm{U}(1)$ gauge transformation. The component fields in the gaugino superfield can be expressed as

$$
\begin{aligned}
W_{\alpha} \mid & =-\frac{1}{4} \bar{D}^{2} D_{\alpha} V \mid=-i \lambda_{\alpha}, \\
D_{\alpha} W_{\beta} \mid & =-\frac{1}{4} D_{\alpha} \bar{D}^{2} D_{\beta} V \mid=-i\left(\sigma^{m n} \epsilon\right)_{\alpha \beta} F_{m n}-\epsilon_{\alpha \beta} D
\end{aligned}
$$

where $\left(\sigma^{m n} \epsilon\right)_{\alpha \beta}=\frac{1}{4}\left(\sigma_{\alpha \dot{\gamma}}^{m} \bar{\sigma}^{n \dot{\gamma} \delta}-\sigma_{\alpha \dot{\gamma}}^{n} \bar{\sigma}^{m \dot{\gamma} \delta}\right) \epsilon_{\delta \beta}$ and $F_{m n}=\partial_{m} A_{n}-\partial_{n} A_{m}$ is the field strength of the gauge field. Alternatively, $F_{m n}$ and $\boldsymbol{D}$ can be expressed as

$$
\begin{aligned}
F_{m n} & =\frac{1}{2 i}\left(\left(\sigma_{m n}\right)_{\alpha}^{\beta} D^{\alpha} W_{\beta}-\left(\bar{\sigma}_{m n}\right)^{\dot{\alpha}}{ }_{\dot{\beta}} \bar{D}_{\dot{\alpha}} \bar{W}^{\dot{\beta}}\right) \mid, \\
\boldsymbol{D} & =-\frac{1}{2} D^{\alpha} W_{\alpha}\left|=-\frac{1}{2} \bar{D}_{\dot{\alpha}} \bar{W}^{\dot{\alpha}}\right| .
\end{aligned}
$$

Note that there are other component fields in $V: V\left|=C, D_{\alpha} V\right|=\chi_{\alpha}, \bar{D}^{\dot{\alpha}} V \mid=\bar{\chi}^{\dot{\alpha}}$, $-\frac{1}{4} D^{2} V\left|=M,-\frac{1}{4} \bar{D}^{2} V\right|=N$. These components can be fixed to zero by imposing the Wess-Zumino gauge fixing condition, and do not appear in component actions. The ordinary kinetic term for the gauge superfield is given by (up to total derivative)

$$
\mathcal{L}=\frac{1}{4 g^{2}} \int d^{2} \theta W^{2}+\text { h.c. }=\frac{1}{4 g^{2}} J+\text { h.c. }=-\frac{1}{4 g^{2}} F^{m n} F_{m n}+\frac{1}{2 g^{2}} \boldsymbol{D}^{2}-\frac{i}{g^{2}} \lambda^{\alpha}\left(\bar{\sigma}^{m}\right)_{\alpha \dot{\beta}} \partial_{m} \bar{\lambda}^{\dot{\beta}},
$$

where $\int d^{2} \theta=-\frac{1}{4} D^{2}$, and

$$
J:=-\frac{1}{4} D^{2} W^{2} \mid=-\frac{1}{2} F^{m n} F_{m n}+\frac{i}{2} F_{m n} \tilde{F}^{m n}+\boldsymbol{D}^{2}-2 i \lambda^{\alpha}\left(\bar{\sigma}^{m}\right)_{\alpha \dot{\beta}} \partial_{m} \bar{\lambda}^{\dot{\beta}} .
$$

Open Access. This article is distributed under the terms of the Creative Commons Attribution License (CC-BY 4.0), which permits any use, distribution and reproduction in any medium, provided the original author(s) and source are credited.

\section{References}

[1] M. Ostrogradsky, Mémoires sur les équations différentielles, relatives au problème des isopérimètres (in French), Mem. Acad. St. Petersbourg 6 (1850) 385 [INSPIRE]. 
[2] R.P. Woodard, Avoiding dark energy with $1 / r$ modifications of gravity, Lect. Notes Phys. 720 (2007) 403 [astro-ph/0601672] [INSPIRE].

[3] G.W. Horndeski, Second-order scalar-tensor field equations in a four-dimensional space, Int. J. Theor. Phys. 10 (1974) 363 [InSPIRE].

[4] T. Kobayashi, M. Yamaguchi and J. Yokoyama, Generalized G-inflation: inflation with the most general second-order field equations, Prog. Theor. Phys. 126 (2011) 511 [arXiv:1105.5723] [INSPIRE].

[5] G. Tasinato, Cosmic acceleration from Abelian symmetry breaking, JHEP 04 (2014) 067 [arXiv:1402.6450] [INSPIRE].

[6] L. Heisenberg, Generalization of the Proca action, JCAP 05 (2014) 015 [arXiv:1402.7026] [INSPIRE].

[7] E. Allys, P. Peter and Y. Rodriguez, Generalized Proca action for an Abelian vector field, JCAP 02 (2016) 004 [arXiv: 1511.03101] [INSPIRE].

[8] S.J. Gates, Jr., Why auxiliary fields matter: the strange case of the $4 D, N=1$ supersymmetric QCD effective action, Phys. Lett. B 365 (1996) 132 [hep-th/9508153] [INSPIRE].

[9] S.J. Gates, Jr., Why auxiliary fields matter: the strange case of the $4 D, N=1$ supersymmetric QCD effective action. 2, Nucl. Phys. B 485 (1997) 145 [hep-th/9606109] [INSPIRE].

[10] S.J. Gates, Jr., M.T. Grisaru, M.E. Knutt and S. Penati, The superspace WZNW action for $4 D, N=1$ supersymmetric QCD, Phys. Lett. B 503 (2001) 349 [hep-ph/0012301] [INSPIRE].

[11] S.J. Gates, Jr., M.T. Grisaru, M.E. Knutt, S. Penati and H. Suzuki, Supersymmetric gauge anomaly with general homotopic paths, Nucl. Phys. B 596 (2001) 315 [hep-th/0009192] [INSPIRE].

[12] S.J. Gates, Jr., M.T. Grisaru and S. Penati, Holomorphy, minimal homotopy and the $4 D$, $N=1$ supersymmetric Bardeen-Gross-Jackiw anomaly, Phys. Lett. B 481 (2000) 397 [hep-th/0002045] [INSPIRE].

[13] D. Nemeschansky and R. Rohm, Anomaly constraints on supersymmetric effective Lagrangians, Nucl. Phys. B 249 (1985) 157 [inSPIRE].

[14] M. Nitta, A note on supersymmetric WZW term in four dimensions, Mod. Phys. Lett. A 15 (2000) 2327 [hep-th/0101166] [INSPIRE].

[15] E.A. Bergshoeff, R.I. Nepomechie and H.J. Schnitzer, Supersymmetric Skyrmions in four-dimensions, Nucl. Phys. B 249 (1985) 93 [INSPIRE].

[16] L. Freyhult, The supersymmetric extension of the Faddeev model, Nucl. Phys. B 681 (2004) 65 [hep-th/0310261] [INSPIRE].

[17] I. Antoniadis, E. Dudas and D.M. Ghilencea, Supersymmetric models with higher dimensional operators, JHEP 03 (2008) 045 [arXiv: 0708.0383] [INSPIRE].

[18] T. Fujimori, M. Nitta and Y. Yamada, Ghostbusters in higher derivative supersymmetric theories: who is afraid of propagating auxiliary fields?, JHEP 09 (2016) 106 [arXiv: 1608.01843] [INSPIRE]. 
[19] J. Khoury, J.-L. Lehners and B. Ovrut, Supersymmetric $P(X, \phi)$ and the ghost condensate, Phys. Rev. D 83 (2011) 125031 [arXiv:1012.3748] [InSPIRE].

[20] J. Khoury, J.-L. Lehners and B.A. Ovrut, Supersymmetric Galileons, Phys. Rev. D 84 (2011) 043521 [arXiv: 1103.0003] [InSPIRE].

[21] M. Koehn, J.-L. Lehners and B.A. Ovrut, Higher-derivative chiral superfield actions coupled to $N=1$ supergravity, Phys. Rev. D 86 (2012) 085019 [arXiv:1207.3798] [INSPIRE].

[22] M. Koehn, J.-L. Lehners and B. Ovrut, Ghost condensate in $N=1$ supergravity, Phys. Rev. D 87 (2013) 065022 [arXiv:1212.2185] [INSPIRE].

[23] I.L. Buchbinder, S. Kuzenko and Z. Yarevskaya, Supersymmetric effective potential: superfield approach, Nucl. Phys. B 411 (1994) 665 [INSPIRE].

[24] I.L. Buchbinder, S.M. Kuzenko and A. Yu. Petrov, Superfield chiral effective potential, Phys. Lett. B 321 (1994) 372 [INSPIRE].

[25] A.T. Banin, I.L. Buchbinder and N.G. Pletnev, On quantum properties of the four-dimensional generic chiral superfield model, Phys. Rev. D 74 (2006) 045010 [hep-th/0606242] [INSPIRE].

[26] S.M. Kuzenko and S.J. Tyler, The one-loop effective potential of the Wess-Zumino model revisited, JHEP 09 (2014) 135 [arXiv: 1407.5270] [INSPIRE].

[27] F. Farakos and A. Kehagias, Emerging potentials in higher-derivative gauged chiral models coupled to $N=1$ supergravity, JHEP 11 (2012) 077 [arXiv:1207.4767] [INSPIRE].

[28] A. Nicolis, R. Rattazzi and E. Trincherini, The Galileon as a local modification of gravity, Phys. Rev. D 79 (2009) 064036 [arXiv:0811.2197] [InSPIRE].

[29] S. Sasaki, M. Yamaguchi and D. Yokoyama, Supersymmetric DBI inflation, Phys. Lett. B 718 (2012) 1 [arXiv:1205.1353] [InSPIRE].

[30] S. Aoki and Y. Yamada, Inflation in supergravity without Kähler potential, Phys. Rev. D 90 (2014) 127701 [arXiv:1409.4183] [InSPIRE].

[31] S. Aoki and Y. Yamada, Impacts of supersymmetric higher derivative terms on inflation models in supergravity, JCAP 07 (2015) 020 [arXiv:1504.07023] [INSPIRE].

[32] C. Adam, J.M. Queiruga, J. Sanchez-Guillen and A. Wereszczynski, Extended supersymmetry and BPS solutions in baby Skyrme models, JHEP 05 (2013) 108 [arXiv: 1304.0774] [INSPIRE].

[33] C. Adam, J.M. Queiruga, J. Sanchez-Guillen and A. Wereszczynski, $N=1$ supersymmetric extension of the baby Skyrme model, Phys. Rev. D 84 (2011) 025008 [arXiv:1105.1168] [INSPIRE].

[34] M. Nitta and S. Sasaki, BPS states in supersymmetric chiral models with higher derivative terms, Phys. Rev. D 90 (2014) 105001 [arXiv:1406.7647] [INSPIRE].

[35] M. Nitta and S. Sasaki, Classifying BPS states in supersymmetric gauge theories coupled to higher derivative chiral models, Phys. Rev. D 91 (2015) 125025 [arXiv:1504.08123] [INSPIRE].

[36] S. Bolognesi and W. Zakrzewski, Baby Skyrme model, near-BPS approximations and supersymmetric extensions, Phys. Rev. D 91 (2015) 045034 [arXiv: 1407.3140] [InSPIRE].

[37] J.M. Queiruga, Baby Skyrme model and fermionic zero modes, Phys. Rev. D 94 (2016) 065022 [arXiv: 1606.02869] [InSPIRE]. 
[38] S.B. Gudnason, M. Nitta and S. Sasaki, A supersymmetric Skyrme model, JHEP 02 (2016) 074 [arXiv: 1512.07557] [INSPIRE].

[39] S.B. Gudnason, M. Nitta and S. Sasaki, Topological solitons in the supersymmetric Skyrme model, JHEP 01 (2017) 014 [arXiv:1608.03526] [INSPIRE].

[40] J.M. Queiruga, Skyrme-like models and supersymmetry in $3+1$ dimensions, Phys. Rev. D 92 (2015) 105012 [arXiv:1508.06692] [InSPIRE].

[41] J.M. Queiruga and A. Wereszczynski, Non-uniqueness of the supersymmetric extension of the $O(3) \sigma$-model, arXiv: 1703.07343 [INSPIRE].

[42] M. Eto, T. Fujimori, M. Nitta, K. Ohashi and N. Sakai, Higher derivative corrections to non-Abelian vortex effective theory, Prog. Theor. Phys. 128 (2012) 67 [arXiv:1204.0773] [INSPIRE].

[43] M. Nitta and S. Sasaki, Higher derivative corrections to manifestly supersymmetric nonlinear realizations, Phys. Rev. D 90 (2014) 105002 [arXiv:1408.4210] [INSPIRE].

[44] M. Nitta, S. Sasaki and R. Yokokura, Supersymmetry breaking in spatially modulated vacua, arXiv: 1706.05232 [INSPIRE].

[45] M. Nitta, S. Sasaki and R. Yokokura, Spatially modulated vacua in relativistic field theories, arXiv: 1706.02938 [INSPIRE].

[46] F. Farakos, C. Germani and A. Kehagias, On ghost-free supersymmetric galileons, JHEP 11 (2013) 045 [arXiv:1306.2961] [INSPIRE].

[47] F. Farakos, C. Germani, A. Kehagias and E.N. Saridakis, A new class of four-dimensional $N=1$ supergravity with non-minimal derivative couplings, JHEP 05 (2012) 050 [arXiv: 1202.3780] [INSPIRE].

[48] T. Kimura, A. Mazumdar, T. Noumi and M. Yamaguchi, Nonlocal $N=1$ supersymmetry, JHEP 10 (2016) 022 [arXiv: 1608.01652] [INSPIRE].

[49] S. Cecotti and S. Ferrara, Supersymmetric Born-Infeld Lagrangians, Phys. Lett. B 187 (1987) 335 [INSPIRE].

[50] J. Bagger and A. Galperin, A new Goldstone multiplet for partially broken supersymmetry, Phys. Rev. D 55 (1997) 1091 [hep-th/9608177] [INSPIRE].

[51] M. Roček and A.A. Tseytlin, Partial breaking of global D $=4$ supersymmetry, constrained superfields and three-brane actions, Phys. Rev. D 59 (1999) 106001 [hep-th/9811232] [INSPIRE].

[52] S.M. Kuzenko and S.A. McCarthy, Nonlinear selfduality and supergravity, JHEP 02 (2003) 038 [hep-th/0212039] [INSPIRE].

[53] S.M. Kuzenko and S.A. McCarthy, On the component structure of $N=1$ supersymmetric nonlinear electrodynamics, JHEP 05 (2005) 012 [hep-th/0501172] [INSPIRE].

[54] H. Abe, Y. Sakamura and Y. Yamada, Matter coupled Dirac-Born-Infeld action in four-dimensional $N=1$ conformal supergravity, Phys. Rev. D 92 (2015) 025017 [arXiv: 1504.01221] [INSPIRE].

[55] S. Cecotti, S. Ferrara and L. Girardello, Structure of the scalar potential in general $N=1$ higher derivative supergravity in four-dimensions, Phys. Lett. B 187 (1987) 321 [INSPIRE].

[56] F. Farakos, S. Ferrara, A. Kehagias and M. Porrati, Supersymmetry breaking by higher dimension operators, Nucl. Phys. B 879 (2014) 348 [arXiv: 1309.1476] [INSPIRE]. 
[57] E. Dudas and D.M. Ghilencea, Effective operators in SUSY, superfield constraints and searches for a UV completion, JHEP 06 (2015) 124 [arXiv: 1503.08319] [INSPIRE].

[58] S.M. Kuzenko and S. Theisen, Supersymmetric duality rotations, JHEP 03 (2000) 034 [hep-th/0001068] [INSPIRE].

[59] S.M. Kuzenko and S. Theisen, Nonlinear selfduality and supersymmetry, Fortsch. Phys. 49 (2001) 273 [hep-th/0007231] [INSPIRE].

[60] S.M. Kuzenko, The Fayet-Iliopoulos term and nonlinear self-duality, Phys. Rev. D 81 (2010) 085036 [arXiv:0911.5190] [INSPIRE].

[61] E.J.S. Villasenor, Higher derivative fermionic field theories, J. Phys. A 35 (2002) 6169 [hep-th/0203197] [INSPIRE].

[62] R. Kimura, Y. Sakakihara and M. Yamaguchi, Ghost free systems with coexisting bosons and fermions, Phys. Rev. D 96 (2017) 044015 [arXiv:1704.02717] [InSPIRE].

[63] J. Wess and J. Bagger, Supersymmetry and supergravity, Princeton Univ. Pr., Princeton U.S.A., (1992) [INSPIRE].

[64] H. Abe, Y. Sakamura and Y. Yamada, Massive vector multiplet inflation with Dirac-Born-Infeld type action, Phys. Rev. D 91 (2015) 125042 [arXiv:1505.02235] [INSPIRE].

[65] S.M. Kuzenko and S.J. Tyler, Complex linear superfield as a model for Goldstino, JHEP 04 (2011) 057 [arXiv: 1102.3042] [INSPIRE].

[66] F. Farakos and R. von Unge, Complex linear effective theory and supersymmetry breaking vacua, Phys. Rev. D 91 (2015) 045024 [arXiv: 1403.0935] [INSPIRE].

[67] D. Ciupke, J. Louis and A. Westphal, Higher-derivative supergravity and moduli stabilization, JHEP 10 (2015) 094 [arXiv: 1505.03092] [INSPIRE].

[68] B.J. Broy, D. Ciupke, F.G. Pedro and A. Westphal, Starobinsky-type inflation from $\alpha^{\prime}$-corrections, JCAP 01 (2016) 001 [arXiv: 1509.00024] [INSPIRE].

[69] A. Maleknejad and M.M. Sheikh-Jabbari, Gauge-flation: inflation from non-Abelian gauge fields, Phys. Lett. B 723 (2013) 224 [arXiv:1102.1513] [inSPIRE].

[70] A. Maleknejad and M.M. Sheikh-Jabbari, Non-Abelian gauge field inflation, Phys. Rev. D 84 (2011) 043515 [arXiv:1102.1932] [INSPIRE].

[71] A. Maleknejad, M.M. Sheikh-Jabbari and J. Soda, Gauge fields and inflation, Phys. Rept. 528 (2013) 161 [arXiv:1212.2921] [INSPIRE].

[72] J. Bagger and A. Galperin, The tensor Goldstone multiplet for partially broken supersymmetry, Phys. Lett. B 412 (1997) 296 [hep-th/9707061] [INSPIRE].

[73] S. Aoki and Y. Yamada, DBI action of real linear superfield in $4 D N=1$ conformal supergravity, JHEP 06 (2016) 168 [arXiv:1603.06770] [INSPIRE]. 